\title{
Quantitative 31P NMR Analysis of Lignins and Tannins
}

\author{
Dimitris S. Argyropoulos ${ }^{2}$, Nicolò Pajer ${ }^{1}$, Claudia Crestini ${ }^{1}$ \\ ${ }^{1}$ Department of Molecular Sciences and Nanosystems, Ca' Foscari University of Venezia ${ }^{2}$ Departments of Chemistry and Forest Biomaterials, North \\ Carolina State University
}

\section{Corresponding Authors}

Dimitris S. Argyropoulos

dsargyro@ncsu.edu

Claudia Crestini

claudia.crestini@unive.it

\section{Citation}

Argyropoulos, D.S., Pajer, N.,

Crestini, C. Quantitative ${ }^{31} \mathrm{P}$ NMR

Analysis of Lignins and Tannins. J. Vis.

Exp. (174), e62696, doi:10.3791/62696 (2021).

\section{Date Published}

August 2, 2021

DOI

$10.3791 / 62696$

URL

jove.com/video/62696

\section{Abstract}

The development of sustainable biorefinery products is confronted, among others, with the challenge of lignin and tannin valorization. These abundant, renewable aromatic biopolymers have not been widely exploited due to their inherent structural complexity and high degrees of variability and species diversity. The lack of a defined primary structure for these polyphenols is further compounded with complex chemical alterations induced during processing, eventually imparting a large variety of structural features of extreme significance for any further utilization efforts.

Consequently, a protocol for the rapid, simple, and unequivocal identification and quantification of the various functional groups present in natural polyphenols, is a fundamental prerequisite for understanding and accordingly tailor their reactivity and eventual utility.

Quantitative ${ }^{31} \mathrm{P}$ NMR offers the opportunity to rapidly and reliably identify unsubstituted, o-mono substituted, and o-disubstituted phenols, aliphatic $\mathrm{OHs}$, and carboxylic acid moieties in lignins and tannins with broad application potential.

The methodology consists of an in situ quantitative lignin or tannin labeling procedure using a suitable ${ }^{31} \mathrm{P}$ containing probe, followed by the acquisition of a quantitative ${ }^{31} \mathrm{P}$ NMR spectrum in the presence of an internal standard. The high natural abundance of the ${ }^{31} \mathrm{P}$ nucleus allows for small amounts of the sample $(\sim 30 \mathrm{mg})$ and short NMR acquisition times ( 30-120 min) with well-resolved ${ }^{31} \mathrm{P}$ signals that are highly dependent on the surrounding chemical environment of the labeled $\mathrm{OH}$ groups.

\section{Introduction}

This procedure, which was recently published in Nature Protocols ${ }^{1}$ has been cited over 3,000 times in the archival literature and has become a routine measurement for lignin and tannin characterization since it provides essential, rapid, and reproducible structural information.

\section{Lignin and tannins}


When Green Chemistry was introduced by Paul T. Anastas and John $\mathrm{C}$. Werner ${ }^{2,3}$, it drastically changed the general conception of Chemistry. In particular, the importance of employing sustainable materials instead of fossil feedstocks, such as oil and coal, as a starting point is highlighted as a crucial aspect ${ }^{2,3}$. Among the different types of biomass, lignin is the most abundant aromatic biopolymer and can be seen as a potential source for industrial commodities and high-added value products ${ }^{4}$.

Lignin is the second most abundant wood-constituent (with cellulose being first and hemicellulose third). Its content in plants varies depending on the plant-type: for example hardwoods characterized by a lower amount of lignin compared to softwoods ( $20 \% \pm 4 \%$ vs. $28 \% \pm 4 \%$ ). In addition, lignin distribution within vegetable tissue is not homogeneous: the higher lignin content can be found in the cell wall ${ }^{5,6}$. Lignin is a polyphenolic material industrially obtained as a byproduct of the paper/cellulose industry ${ }^{7}$. It is recovered from the wood pulping process, in which wood chips are primarily processed in the presence of $\mathrm{OH}^{-}$and/or $\mathrm{OH}^{-}+\mathrm{HS}^{-}$ion conditions to separate cellulose from hemicellulose and lignin (Soda and/or Kraft processes) $^{8,9}$.

The first attempts to study lignin were made by Payen and Schultze, respectively, in 1838 and $1865^{10}$. In 1977, Adler summarized all the relevant available knowledge of that time ${ }^{11}$. It is currently recognized that the lignin building blocks are three phenyl-propanoid units: $p$-coumaryl, coniferyl, and sinapyl alcohols. These monomers, thanks to a free radical polymerization process, give rise to $p$ hydroxyphenyl, guaiacyl, and sinapyl units that eventually broadly constitute lignin (Figure 1$)^{12}$. The lack of a primary structure in lignins implies an inherent difficulty for its structural characterization. Accordingly, the evaluation of the distribution of molecular weight has always been somewhat controversial. Milled wood lignin, the lignin isolated under mild conditions that approximate mostly protolignin ${ }^{10}$, is composed of oligomers ${ }^{13}$ which highly interact via supramolecular aggregation processes ${ }^{14,15}$. 


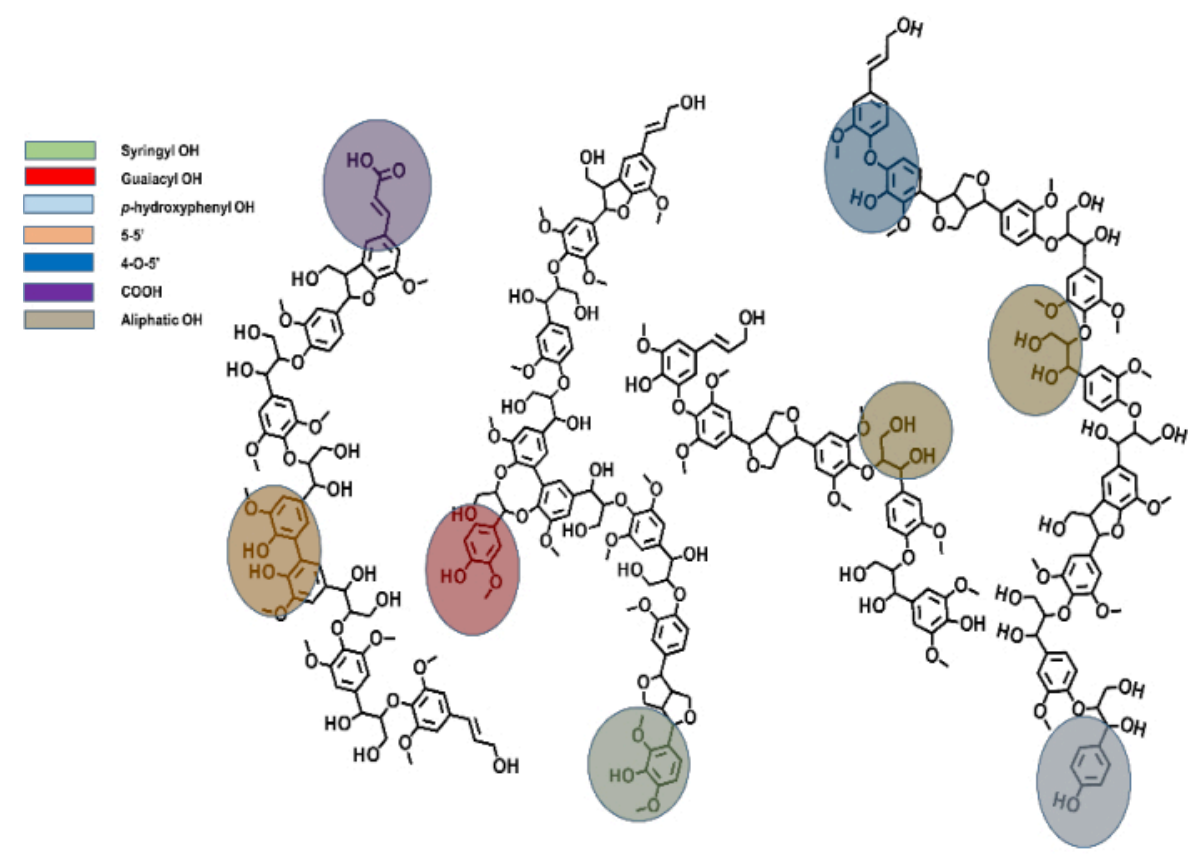

Figure 1: A representative model of softwood lignin in which the different types of bonds are highlighted. Please click here to view a larger version of this figure.

Lignins are commonly classified depending on: (a) the type of wood from which they are derived (e.g., hardwood and softwood), (b) the process used to isolate it. The most crucial industrial lignin types are Kraft, Lignosulfonates, and Organosolv.

The structure of lignin is highly dependent upon its origin and processing chemistry. More specifically, when the rather complex and irregular structure of lignin is compounded with its natural diversity and the complex processing chemistries, a material of extreme variability, diversity, and heterogeneity emerges, limiting its use to low-value applications ${ }^{16}$. While softwood lignins contain mainly guaiacyl units $(G)$ with negligible amounts of $p$-hydroxyphenyl groups (G lignin), hardwood lignins are composed by guaiacyl and syringyl subunits (GS lignin) in varying ratios and grass lignins are constituted by guaiacyl, syringyl, and $p$-hydroxyphenyl (GSH lignin) subunits. The extractive approach used for isolation dramatically affects the structure of the emerging $\operatorname{lignin}^{17}$. Figure 2 depicts three lignin structures, differing by the isolation approach employed. Some considerations regarding the effect of the extraction method could be highlighted. Firstly, Kraft lignin is a dealkylated, highly fragmented, and condensed lignin, while Organosolv lignin has a structure similar to milled wood lignin (isolated using the Bjorkman approach) ${ }^{18,19,20}$. Finally, lignosulfonates are characterized by a high degree of sulfonation, depending on the intensity and the conditions of the extractive sulfonation process. 


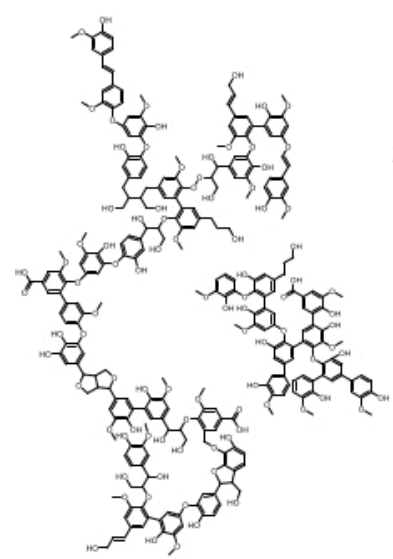

A

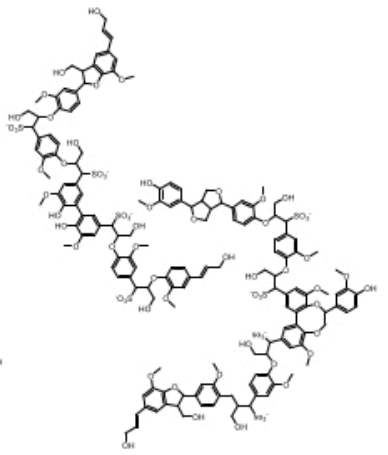

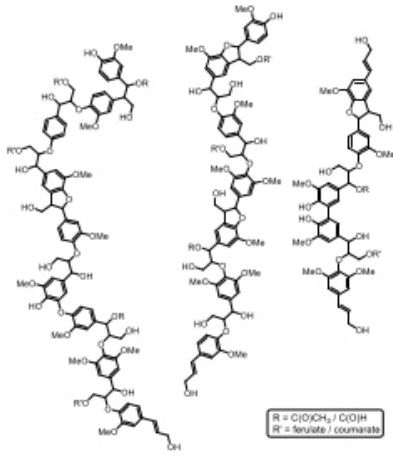

C

Figure 2: Representative structures for technical lignins. In this figure, the differences among the different types of lignin can be seen. (A) Softwood Kraft lignin is highly condensed, (B) lignosulfonates are characterized by sulfonic groups on saturated carbons, and $(\mathbf{C})$ organosolv lignin has a structure similar to the one of milled wood lignin. Please click here to view a larger version of this figure.

Similar to lignins, tannins are polyphenolic compounds that are found in plants. A recent and updated review on tannins' extractive approaches and applications was recently released by Das et al. ${ }^{21}$. The importance of tannins in everyday life can be highlighted considering two examples: they impart taste and color to wines ${ }^{22}$; furthermore their poly-phenolic structure offers antioxidant characteristics and makes them ideal for application in the tanning industry ${ }^{23}$. Tannins are divided into two classes: hydrolyzable and non-hydrolyzable. Hydrolyzable tannins can be considered a polymer of gallic, di-gallic, and ellagic acid esters (Figure 3). These esters result from the esterification of the phenolic acids with sugar molecules (e.g., glucose, rhamnose, and arabinose). 

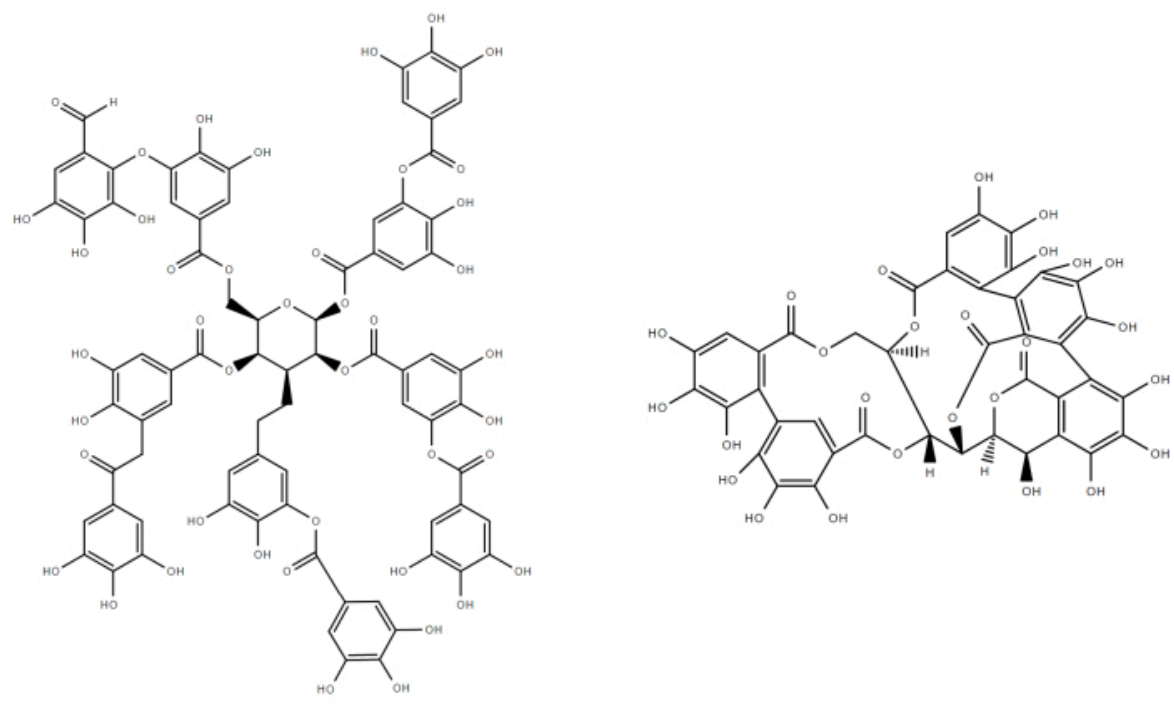

Figure 3: Typical hydrolysable tannins: tannic acid, vescalgin. Please click here to view a larger version of this figure.

Non-hydrolyzable tannins, also known as condensed tannins, are polymers and oligomers deriving from flavan-3-ols. Among flavan-3-ols, catechins and gallocatechin are the most frequent. They are colorless crystalline compounds (Figure
4). The polymerization creates a polymer characterized by a helicoidal structure. The aromatic hydroxy groups are directed on the exterior of the helix, while the pyran oxygens are in the interior. 


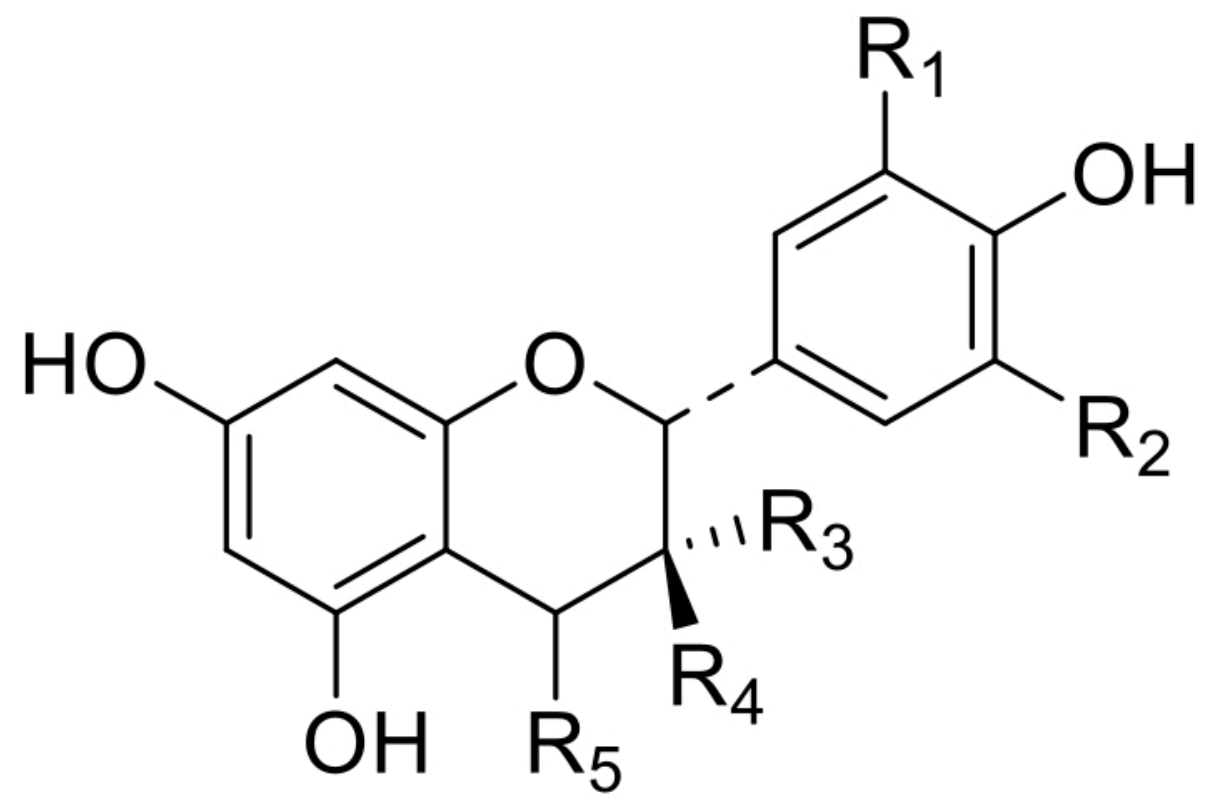

Figure 4: Proantocyanidin structures: $\mathbf{R}=\mathbf{H}, \mathbf{O H}, \mathbf{O} \mathbf{C H}_{3}$. Please click here to view a larger version of this figure.

\section{Characterization of lignins and tannins using NMR}

Two types of information are crucial in lignin or tannin characterization: (a) chemical structure (e.g., hydroxy group content, nature, and frequency of interunit linkages) and (b) molecular weight and polydispersity. Since the early studies on lignin, different techniques have been employed to achieve these goals, and two classes of methods have emerged: chemical and physical methods.

In lignin chemistry, chemical methods, such as alkaline nitrobenzene oxidation, derivatization followed by reductive cleavage, permanganate oxidation, and thioacidolysis, have been historically widely used $24,25,26,27,28,29$. However, even if the analytical protocols have been implemented and optimized, they are time-demanding, laborious, and require extensive experimental skills ${ }^{30}$. Alternatively, from the beginning of the instrumental analysis, physical methods have been used to perform lignin and tannin characterizations ${ }^{31}$. These techniques allow overcoming the problems of classical methods making it easy to characterize lignin structure.

Nuclear Magnetic Resonance (NMR) allows obtaining information about lignin structure and chemical composition among the instrumental techniques. In particular, data from quantitative monodimensional ${ }^{1} \mathrm{H}$ NMR spectra and quantitative ${ }^{13} \mathrm{C}$ NMR spectra can provide information about different types of lignin interunit bondings $32,33,34,35$. Unfortunately, monodimensional spectra suffer from signal overlap, which can seriously undermine signal integration efforts. Quantitative versions of HSQC (Heteronuclear Single Quantum Coherence), Q-HSQC (Quantitative Heteronuclear Single Quantum Coherence), have been used to understand lignin structure better, providing helpful information about internal linkages. However, they cannot be 
fully utilized to determine the various buildings units ${ }^{13,36,37}$ quantitatively.

To overcome the issues associated with mono- and two-dimensional NMR, substrate derivatization has been considered. Among the advantages of this approach is that specific labels can be introduced within the complex macromolecule and no spectral interference results from the solvent in which the labeled substrates are dissolved ${ }^{1}$. Verkade was the pioneer in this field, performing ${ }^{31} \mathrm{P}$ NMR analysis of phosphorous derivatives, coal derivatives, and related compounds ${ }^{38}$. In its publication, a screening of different phosphorus-containing reagents (phospholanes) was performed, and the chemical shift of other labeled compounds was recorded. Argyropoulos' team first introduced derivatization for the quantitative and qualitative analysis of hydroxy groups in lignin in 1991. After studying the derivatization of lignin model compounds using phosphorus-containing reagents, his group paved the way for one of the most daily-used techniques in lignin chemistry, ${ }^{31} \mathrm{P}$ NMR analysis ${ }^{39,40,41,42,43}$. Among the different phospholanes examined, Argyropoulos arrived at the use of 2-chloro-4,4,5,5-tetramethyl-1,3-2-dioxaphospholane (TMDP) as being the most suitable one to perform lignin analysis $^{44}$. TMDP selectively reacts with hydroxy groups causing the quantitative formation of phosphorus-containing derivatives characterized by specific ${ }^{31}$ P NMR chemical shifts (Figure 5).

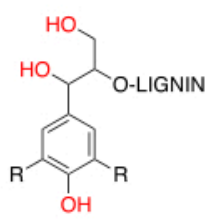

$\mathrm{R}=\mathrm{H}, \mathrm{OCH}_{3}$
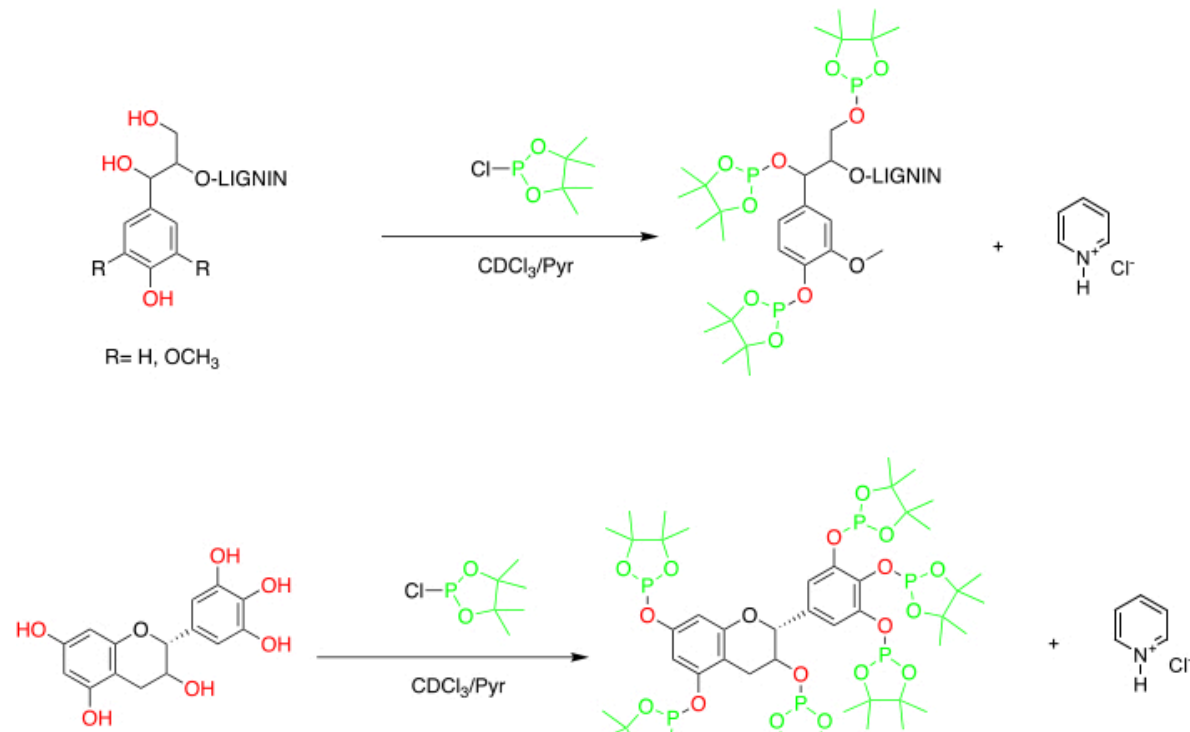

Figure 5: Lignin and tannin phosphytilation chemistry. Labeling lignin and tannin labile $\mathrm{H}$ groups is accomplished by in situ reaction. The labeled polyphenols are characterized by specific ${ }^{31} \mathrm{P}$ NMR bands corresponding to the different type of hydroxy groups. Please click here to view a larger version of this figure. 
Sample derivatization is performed in a pyridine/chloroform (1.6:1) mixture; this choice results from an accurate evaluation. Pyridine has two advantages. Firstly, selecting a solvent characterized by a Hildebrand parameter of about $22.1 \mathrm{MPa}^{1 / 2}$ simplifies and amplifies lignin solubilization ${ }^{45}$. Consequently, the addition of pyridine as a solvent, whose Hildebrand parameter equals 21.7, is thus optimal. Secondly, the reaction of TMDP with hydroxy groups is accompanied by the formation of hydrochloric acid $(\mathrm{HCl})$ as a by-product with concomitant negative implications toward the facile formation of lignin-phospholane derivatives. For this reason, the resulting $\mathrm{HCl}$ needs to be neutralized. When present in significant excess, the basicity of the pyridine, relative to TMDP, allows for the neutralization of the $\mathrm{HCl}$ (via the formation of pyridine hydrochloride).

The use of the recommended pyridine/deuterated chloroform binary solvent system is based on three reasons. Firstly, it favors sample dissolution. Secondly, as pyridine hydrochloride is soluble in chloroform, it can prevent precipitation and deterioration of the final spectrum. Thirdly, deuterated chloroform is chosen for its unique singlet signal, allowing locking of the NMR spectrometer during the acquisition process. Sample derivatization is performed in the presence of an internal standard. In this way, when the sample and the standard are derivatized, the comparison of the integrals of the peaks of the sample and the standard allows the quantification of the amount for each type of hydroxy group present. Various compounds have been considered as internal standards. These compounds are characterized by a single hydroxy group per molecule, offering a single sharp signal in the ${ }^{31} \mathrm{P}$ NMR spectrum after derivatization. The selection of the standard must be made carefully. Its signal should not overlap with those of the derivatized sample. Cholesterol was widely used during the early days. However, a partial overlap with signals arising from aliphatic hydroxy group limits its use. For routine analysis, internal standard solutions of N-hydroxy-5-norbornene-2,3-dicarboximide (NHND) are preferred. However, owing to NHND instability, its standard solutions can be stored only for a few days ${ }^{46}$.

\section{Protocol}

The following flow chart (Figure 6) outlines the whole experimental protocol to perform a ${ }^{31} \mathrm{P}$ NMR analysis of lignins and tannins. 


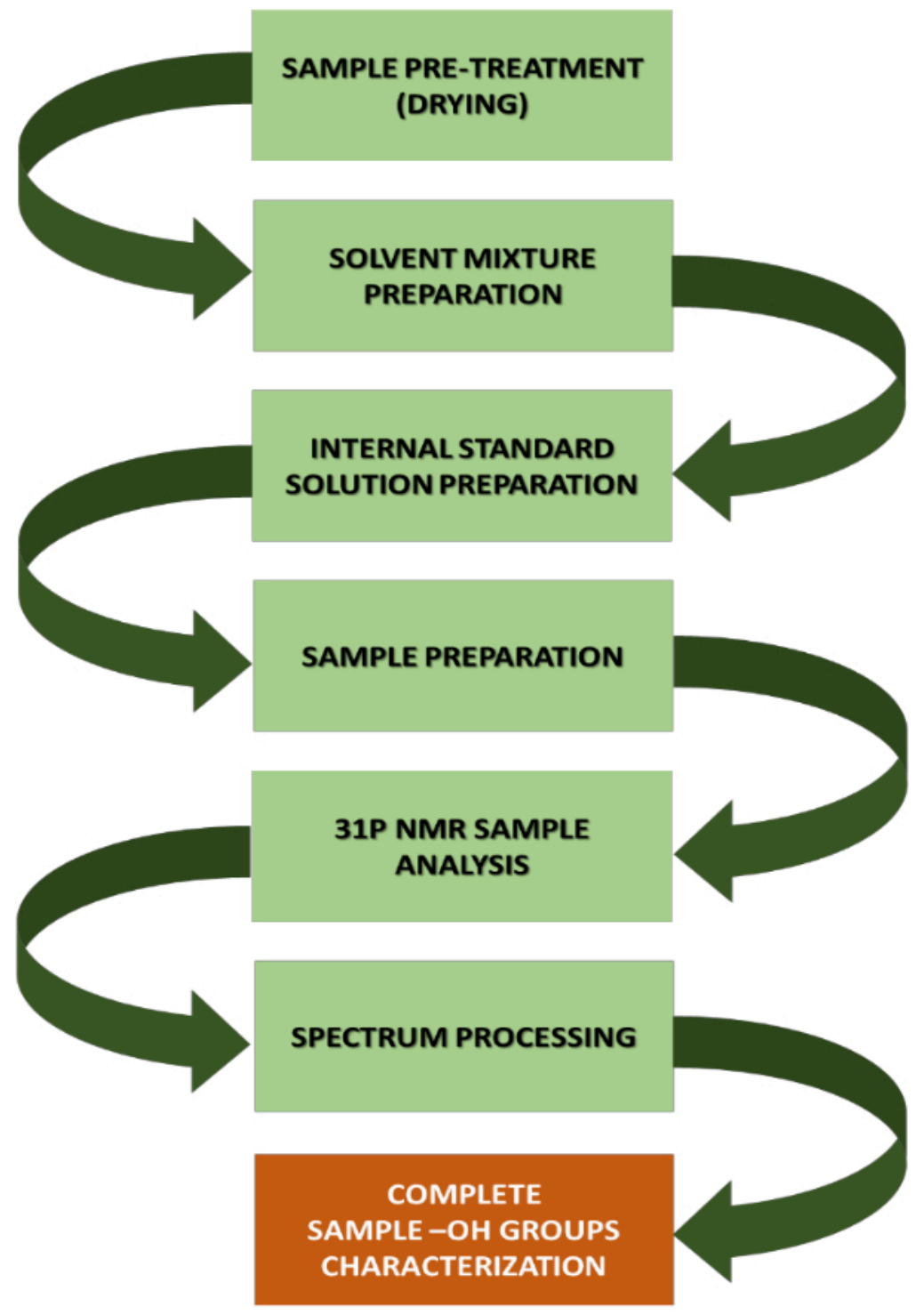

Figure 6: Procedure for the ${ }^{31}$ P NMR analysis of lignins and tannins. Please click here to view a larger version of this figure.

\section{Sample pretreatment}

1. Dry an aliquot (around $100 \mathrm{mg}$ ) of the analyte (lignin or tannin sample) overnight in a vacuum oven set at $40^{\circ} \mathrm{C}$. NOTE: Particular attention is needed on the temperature selection since temperatures higher than $40{ }^{\circ} \mathrm{C}$ may chemically alter the sensitive structure of the examined polyphenols.

2. After drying, rapidly transfer the sample to an anhydrous calcium sulfate desiccator until it reaches room temperature. This step is compulsory to avoid the sample absorbing humidity from the environment. 


\section{Solvent solution preparation}

1. Prepare a pyridine/deuterated chloroform solvent mixture in a $20 \mathrm{~mL}$ sample vial by mixing anhydrous pyridine and deuterated chloroform in a 1.6/1 (v/v) ratio. CAUTION: Pay attention while manipulating pyridine and deuterated chloroform. These compounds are flammable, harmful, and toxic. Prepare and use the solution in a well-ventilated fume-hood using appropriate gloves.

2. Add 5-8 g of well-washed and dried activated $5 \mathrm{~A}$ molecular sieves in $3.2 \mathrm{~mm}$ pellets to remove water traces. In addition, the use of a septum cap is highly recommended to prevent air contact and moisture contamination of the solvent system. Store the prepared solution in the dark.

\section{Internal standard solution (IS) preparation}

1. In a $2 \mathrm{~mL}$ Erlenmeyer flask, prepare a $0.1 \mathrm{M}$ solution of chromium (III) acetylacetonate (about $10 \mathrm{mg}$ ) and internal standard (around $35.8 \mathrm{mg}$ of NHND or $77.3 \mathrm{mg}$ of cholesterol) in the solvent solution previously prepared. CAUTION: Chromium (III) acetylacetonate is harmful; during its manipulation, wear appropriate gloves.

2. Record the exact weight of the IS added in the IS solution.

3. Transfer the IS solution in a vial equipped with a sealed cap containing activated molecular sieves (see point 2.2) and store it in the dark at $40^{\circ} \mathrm{C}$.

\section{NMR sample solution preparation}

1. Accurately weigh $\sim 30 \mathrm{mg}$ of sample in a $2 \mathrm{~mL}$ vial equipped with a stirring bar. Seal the vial with a septum cap.
2. Add $0.5 \mathrm{~mL}$ of the solvent system solution to the sample vial.

3. Transfer $100 \mu \mathrm{L}$ of the IS solution in the sample vial via a micropipette. Magnetically stir the resulting dispersion (500 rpm) until all the lignin or the tannin is dissolved, resulting in a clear solution.

NOTE: Since complete sample solubilization is imperative, this step could take up to $12 \mathrm{~h}$.

4. Transfer $0.1 \mathrm{~mL}$ of TMDP to the sample solution. Place the sample under vigorous magnetic stirring. Keep the sample solution sealed. Use TMDP in a well-ventilated fume-hood while wearing appropriate gloves.

CAUTION: TMDP and its vapors are corrosive, harmful, and rapidly interact with water.

NOTE: The formation of a yellow precipitate is due to water traces in the sample or the pyridine/chloroform solution. In such a case, the procedure must be repeated by ensuring all possible moisture contamination is avoided.

5. Transfer the sample solution into an NMR tube using a Pasteur pipette.

\section{NMR analysis}

1. Load the tube into the NMR instrument. The spectrometer used to perform this analysis needs a broadband probe.

2. Fix the experimental parameters according to the setting shown in Table $1^{1}$. 


\begin{tabular}{|c|c|}
\hline PULSE PROGRAM & Inverse gated decoupling pulse (zgig) \\
\hline NUCLEOUS & $31 \mathrm{P}$ \\
\hline SPECTRAL WIDTH & 100 p.p.m. \\
\hline ACQUISITION TIME & $-0.8 \mathrm{~s}$ \\
\hline RELAXATION DELAY & $\geq 10 \mathrm{~s}$ \\
\hline SCANS NUMBER & 64 or more \\
\hline SPECTRUM CENTER & 140 p.p.m. \\
\hline
\end{tabular}

Table 1: Experimental parameters to record ${ }^{31} \mathrm{P}$ NMR spectra of derivatized lignins or tannins.

3. Set the spectrometer frequency using the resonance frequency of deuterated chloroform, shim the sample and tune the spectrometer. Then, start the acquisition.

\section{Spectrum processing and analysis}

1. Process ${ }^{31} \mathrm{P}$ NMR raw data by an appropriate standard software according to the following steps.

1. Perform Fourier transformation.

2. Adjust Phase by manual phase correction (Processing | Phase Correction | Manual Correction).

3. Correct baseline manually, carefully setting zero points (Processing | Baseline | Multipoint Baseline Correction).
2. Signal calibration.

1. Set the signal for the phosphitylated water at the chemical shift value of $132.2 \mathrm{ppm}$ (Analysis I Reference | Reference).

NOTE: The presence of a sharp ${ }^{31} \mathrm{P}$ signal at 175 ppm is due to the excess of TMDP. Its presence ensures the complete derivatization of the sample. If this peak is absent, one needs to revisit the whole procedure by providing a thorough sample and solvent drying and adding more TMDP. Once this is guaranteed, the spectrum is zoomed in the spectral range 132 to around 150 ppm (Figure 7). 
TMDP excess $=$ complete derivatization of the sample
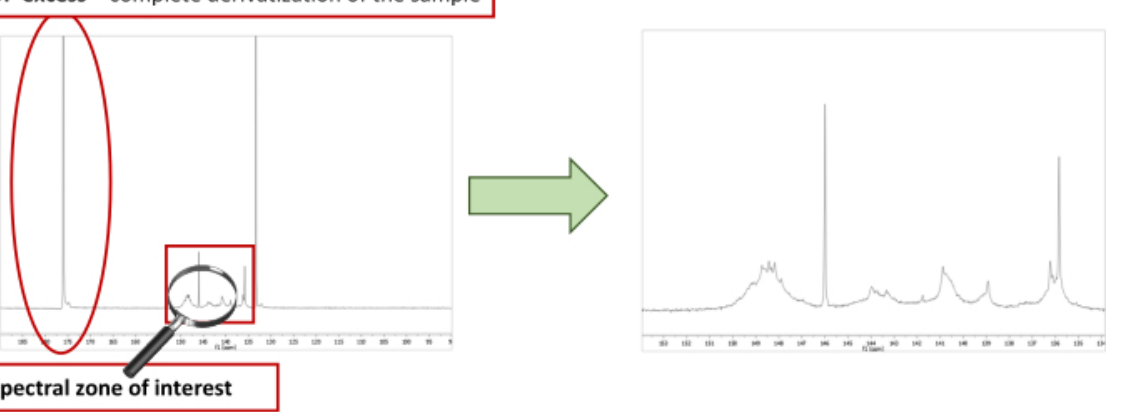

Figure 7: Check the presence of an excess of TMDP: if it can be seen, the derivatization of the sample is complete.

The spectra can then be analyzed. To do that zoom in the spectral range between 155 and 132 ppm. Please click here to view a larger version of this figure.

3. Integration

1. Normalize integration by setting the internal standard to 1.0 (click on the Peak | Edit Integral
| Normalized: 1.00). Perform spectrum integration according to the chemical shifts reported in the following tables. Use Table 2 for lignins and Table 3 for tannins.

\begin{tabular}{|c|c|}
\hline FUNCTIONAL GROUP & CHEMICAL SHIFT (ppm) \\
\hline Aliphatic $\mathrm{OH}$ & $149.0-146.0$ \\
\hline Phenolic $\mathrm{OH}$ & $144.0-137.4$ \\
\hline C5 substituted phenolic OH & $143.0-140.2$ \\
\hline 5-5' phenolic OH & $141.7-140.2$ \\
\hline Syringyl OH & $143.2-142.7$ \\
\hline 4-O-5' OH & $142.8-141.7$ \\
\hline Guaiacyl OH & $140.2-138.8$ \\
\hline p-hydroxyphenyl OH & $138.8-137.4$ \\
\hline COOH & $136.0-133.6$ \\
\hline
\end{tabular}


Table 2: ${ }^{31} \mathrm{P}$ NMR chemical shifts for lignin phosphitylated OH-groups.

\begin{tabular}{|c|c|c|}
\hline \multicolumn{2}{|c|}{ RUNCTIONAL GROUP } & CHEMICAL SHIFT (ppm) \\
\hline \multicolumn{2}{|c|}{ Ring A } \\
\hline o-unsubstituted phenolic & 137.9-137.4 \\
\hline o-substituted PHENOLIC & Ring B & $138.8-137.9$ \\
\hline Catechol OH & $140.2-138.8$ \\
\hline Pyrogallol OH & $144.0-140.2$ \\
\hline \multicolumn{2}{|c|}{ Ring C } \\
\hline AliphatiC OH & & $146.0-145.0$ \\
\hline
\end{tabular}

Table 3: ${ }^{31} \mathrm{P}$ NMR chemical shift for tannin phosphitylated $\mathrm{OH}-\mathrm{groups}$.

NOTE: Using standard spectral-processing software, it is possible to set pre-defined regions of the chemical shift to be integrated. This opportunity is advantageous when several spectra have to be processed.

\section{Functional group quantification}

1. Calculate the concentration of the IS solution.

IS concentration $(M)=\frac{\text { mass of IS }(\mathrm{mg})}{M W_{I S}(\mathrm{~g} / \mathrm{mol})} \cdot \frac{I S p u r i t y(\%)}{100} \cdot \frac{1}{\text { volume of IS solution }(\mathrm{mL})}$

2. Calculate the equivalent amount of the specific signal:

Functional group amount (mmol per gram of lignin $)=$ normalized peak area $I S$ concentration.volume of IS added to the sample $(\mathrm{mL})$ sample (lignin or tannin) weight $(g)$
Functional group amount (mmol per gram of lignin $)=$ $\frac{\text { normalized peak area }}{\text { sample weight }(g)} \cdot$ IS concentration $\cdot 0,1$

\section{Representative Results}

The described protocol can be applied both for the analysis of lignins and tannins. In lignin chemistry, this method is fundamental because it allows the detection and quantification of the different types of hydroxy groups. Figure 8A-D show examples of ${ }^{31} \mathrm{P}$ NMR spectra of lignins and tannins acquired with spectrometers working at different frequencies. The spectrum shown in Figure 8A was recorded using a $300 \mathrm{MHz}$ NMR spectrometer, while Figure 8D was recorded with a $700 \mathrm{MHz}$ NMR instrument. 
A
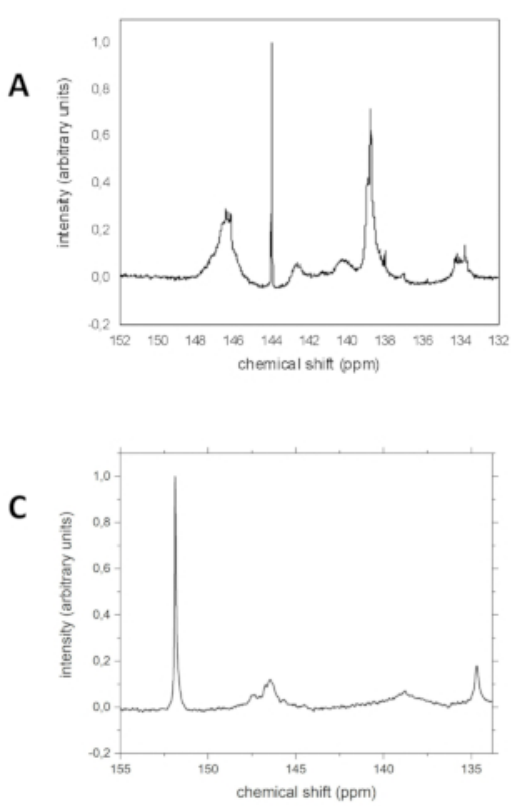

B
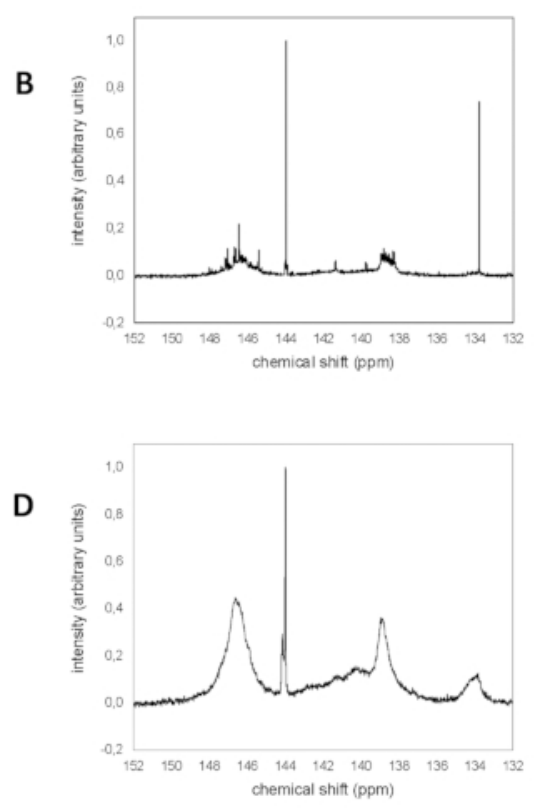

Figure 8: Quantitative ${ }^{31} \mathrm{P}$ NMR spectrum of (A) softwood kraft lignin (spectrum recorded with a 300 MHz spectrometer on $\mathbf{3 0 . 8} \mathbf{~ m g ~ o f ~ l i g n i n ) , ~ ( B ) ~ s o f t w o o d ~ l i g n o s u l f o n i c ~ a c i d ~ ( s p e c t r u m ~ r e c o r d e d ~ w i t h ~ a ~} 300$ MHz spectrometer on $\mathbf{3 0 . 1} \mathrm{mg}$ of lignin after conservation of lignosulfonate to lignosulfonic acid), (C) Acacia tannin (spectrum recorded with a $300 \mathrm{MHz}$ spectrometer on a $30.3 \mathrm{mg}$ sample) and (D) softwood kraft lignin (spectrum recorded with a $700 \mathbf{M H z}$ spectrometer on $\mathbf{7 . 2} \mathbf{~ m g}$ of lignin). Please click here to view a larger version of this figure.

These spectra were carefully recorded and processed manually. The typical signals for aliphatic (150-145 ppm), aromatic (145-137 ppm), and carboxylic (136-134 ppm) hydroxy groups are very well resolved and as such readily integrated. If the spectral window is opened (from 95 to 190 ppm, Figure 8), three sharp, strong peaks (175, 144, and $132 \mathrm{ppm}$ ) are apparent. Those are due to the excess of TMDP, the internal standard (cholesterol or NHND), and the hydroxylated-TMDP (caused by water traces), respectively.
In contrast to kraft and organosolv lignin, lignosulfonates are insoluble in the pyridine/chloroform mixture. To obtain a reliable ${ }^{31} \mathrm{P}$ NMR spectrum, solubility is mandatory. To overcome this issue, lignosulfonates can be converted to the corresponding lignosulfonic acids before derivatization. Treating lignosulfonate solutions with strong acids (i.e., sulfuric acid), or acid exchange resins (e.g., Dowex $1 \mathrm{H}$, a strong acid cation exchanger) drives the conversion of all the sulfonate groups in their acidic forms. The resulting products can be removed from the acidic solution using selective adsorptive resins (XAD-7, a polar adsorbent used to isolate compounds characterized by molecular weights up to 60,000 u.m.a) analyzed using this protocol. Figure 8B shows the quantitative ${ }^{31} \mathrm{P}$ NMR spectrum of a TMDP 
derivatized lignosulfonic acid. Even in this case, the different signals of the hydroxy groups are evident. Figure $\mathbf{8 C}$ shows a typical quantitative ${ }^{31} \mathrm{P}$ NMR spectrum of a tannin sample derivatized using TMDP. A characteristic signal from the different aliphatic $\mathrm{OH}$ (Ring $C)$, pyrogallol, and catechol units in ring $B$ and units in ring $A$ are well visible.

\section{Discussion}

The described method represents the implementation and optimization of the analytical protocol aimed at the qualitative and quantitative characterization of lignins as developed by Argyropoulos $37,38,39,40,41,42$. Compared to many other techniques available for lignin structural elucidation, the method has been widely accepted as being among the most facile, rapid, and reproducible. The validity of the wet chemical methods (e.g., nitrobenzene, permanganate oxidations, etc.) relies on the good experimental skills of the operator, effectively confining the method to limited operators. Furthermore, it is not uncommon to encounter correction factors in the literature for wet chemical methods to account for several drawbacks. The described ${ }^{31} \mathrm{P}$ NMR protocol does not require advanced experimental skills making this readily applicable, user-friendly, and widely available. Compared to other instrumental analytical methods, ${ }^{31} \mathrm{P}$ NMR is the only technique capable of precisely detecting and quantifying the different hydroxy groups in lignins. For instance, FTIR can be used to identify various hydroxy groups such as ${ }^{1} \mathrm{H}$ NMR. Both techniques, however, suffer since they cannot offer reliable quantitative data due to extensive signal overlap issues. Another widely used technique is UV-Vis spectroscopy, firstly reported by Goldschmid. The approach, however, is limited to a general overall determination of hydroxy groups since it cannot effectively differentiate among aliphatic, aromatic, and carboxylic $\mathrm{OHs}^{47}$.
From an economic point of view, the only limitation of the ${ }^{31} \mathrm{P}$ NMR technique is the price of TMDP, which is a relatively expensive reagent. It costs about 190 USD per gram; consequently, if the cost of analysis would be approximated only to the price of TMDP, excluding those deriving from the pyridine/chloroform mixture and those of the operator time, it would amount to about 24 USD per analysis. To solve this issue, many laboratories resort to synthesizing TMDP, thus, reducing the reagent costs. To do this, pinacol and phosphorous trichloride are reacted in the presence of triethylamine ${ }^{44}$. Technically, this reaction is relatively easy; however, care in the use of phosphorous trichloride and its work-up, including well-controlled vacuum distillation, is required. More details as to the synthesis of the TMDP can be supplied upon request.

Even though this protocol is among the best in terms of ease, reproducibility, and precision, some critical points need to be highlighted. Firstly, the sample needs to be fully soluble in the identified pyridine/chloroform mixture. This consideration is fundamental because the quantitative phosphitylation reaction of the hydroxyl groups needs to take place under completely homogeneous conditions. If only part of the sample is solubilized, the resulting analysis would be inaccurate. Secondly, the sample to be examined needs to be moisture- and solvent-free since these variables will detrimentally affect the precision and overall success of the analysis. Traces of humidity will react with TMDP giving 2hydroxy-4,4'-5,5'-tetramethyl-1,3,2-dioxaphospholane. This compound is a pale-yellow flocculating salt, insoluble in the pyridine/chloroform solvent mixture, causing inadequate NMR signal acquisition. Since only a small weight $(\sim 30 \mathrm{mg})$ of a sample is required, it needs to be free of volatiles for its precise weight to be accurately known before the analysis. 
Sometimes, sample solvation issues can be promoted (especially for highly oxidized samples) by adding small amounts of a co-solvent (i.e., dimethylformamide), aiding sample dissolution. In principle, every solvent that does not interact with TMDP can be used to help sample dissolution. The election of a co-solvent cannot include co-solvents containing labile hydroxy or amino groups since they react with the reagent, causing misleading final spectra. Notably, dimethylsulfoxide also reacts with TMDP precluding its use as a co-solvent. Pyridine-based ionic liquids, such as 1-allyl-3butylpyridinium chloride, can be used when solubility issues arise; however, the ionic liquid should be once again $\mathrm{dry}^{48}$. To dissolve lignosulfonates (a lignin type characterized by a high sulfonation degree), a pre-treatment involving the conversion of neutralized groups into their acidic form was demonstrated to be helpful. Lignosulfonates can be conveniently converted into their acidic conditions using acidic exchange resins in aqueous media. The resulting lignosulfonic acids are isolated from the solution by their adsorption on specific resins (e.g., XAD-7) and desorption in ethanol. Evaporation of the ethanolic solutions over reduced pressure at $40{ }^{\circ} \mathrm{C}$ allows the isolation of lignosulfonic acids. These lignins can then be characterized by ${ }^{31} \mathrm{P}$ NMR because they are soluble in the pyridine/chloroform mixture proposed by the protocol.

Prolonged vacuum drying at mild temperatures effectively reduces the amount of moisture and other volatiles in each sample. Notably, small quantities of water do not affect the final spectrum because TMDP is added in excess. In addition, in some cases, a small amount of 2-hydroxy-4,4'-5,5'-tetramethyl-1,3,2-dioxaphospholane may result from the humidity present in the NMR tube or the sample vial. In these cases, stirring is sufficient to dissolve the amount of the formed precipitate completely. If a high amount of 2-hydroxy-4,4'-5,5'-tetramethyl-1,3,2-dioxaphospholane is formed, it is suggested to repeat the sample preparation, improving the drying treatment. For instance, before use, all glassware can be briefly heated with a heat gun.

The spectral range used to record the spectrum is broad compared to the region of interest for the signal regarding the different hydroxyl groups. However, this is compulsory to understand whether the sample derivatization occurred successfully. The confirmation of complete sample derivatization is given by the presence of a strong signal around $174 \mathrm{ppm}$. This sharp peak is due to the unreacted TMDP, and its existence ensures that the reagent was present in excess, and therefore, all hydroxyl groups have been derivatized. If this peak is absent, the two most probable causes are: (1) the amount of TMDP used is insufficient to perform the complete derivatization of the sample, or (2) a high amount of water is present in the sample. In the first case, using a higher amount of TMDP would likely ensure the sample's complete derivatization, and the signal at $174 \mathrm{ppm}$ will appear. In the second case, the sample should be dried more extensively. Once an excess of TMDP is ensured, peak integration can be performed. Before this operation, zoom to a narrower window (150 to $132 \mathrm{ppm}$ ) that confines the signals of interest.

The amount of sample $(\sim 30 \mathrm{mg})$ to be analyzed, reported in the above experimental protocol, has been selected to collect good quality spectra for a $300 \mathrm{MHz}$ NMR spectrometer or more. Nevertheless, we have observed that it is possible to reduce the sample amount if a $500 \mathrm{MHz}$ or higher field magnet is used. For instance, in Figure 8D, the NMR spectrum (resulting from a $700 \mathrm{MHz}$ instrument) of a sample prepared with $7.2 \mathrm{mg}$ of lignin is shown. Signal integration of this spectrum offers the same results as those obtained when using higher amounts of lignin. This fact amplifies the 
applicability of this protocol for all the research in which small quantities of products are available.

Overall, this experimental protocol can be applied to many research and development applications when understanding the origin and fate of the various hydroxy groups present in lignins and tannins is required. In particular, when coupled with GPC and HSQC data, the resulting data offers the opportunity to further elaborate and speculate over the structure of lignin or a tannin. In many instances where chemical modifications are applied to the hydroxy groups of lignin or a tannin, quantitative ${ }^{31} \mathrm{P}$ NMR analyses can be extremely valuable to detect whether these modifications occurred and to which degree. For instance, Figure 9 shows two NMR spectra of the same lignin before and after its oxidation. A simple qualitative evaluation shows the reduction of both aliphatic and aromatic hydroxy groups upon oxidation, thus providing valuable information and guidance.

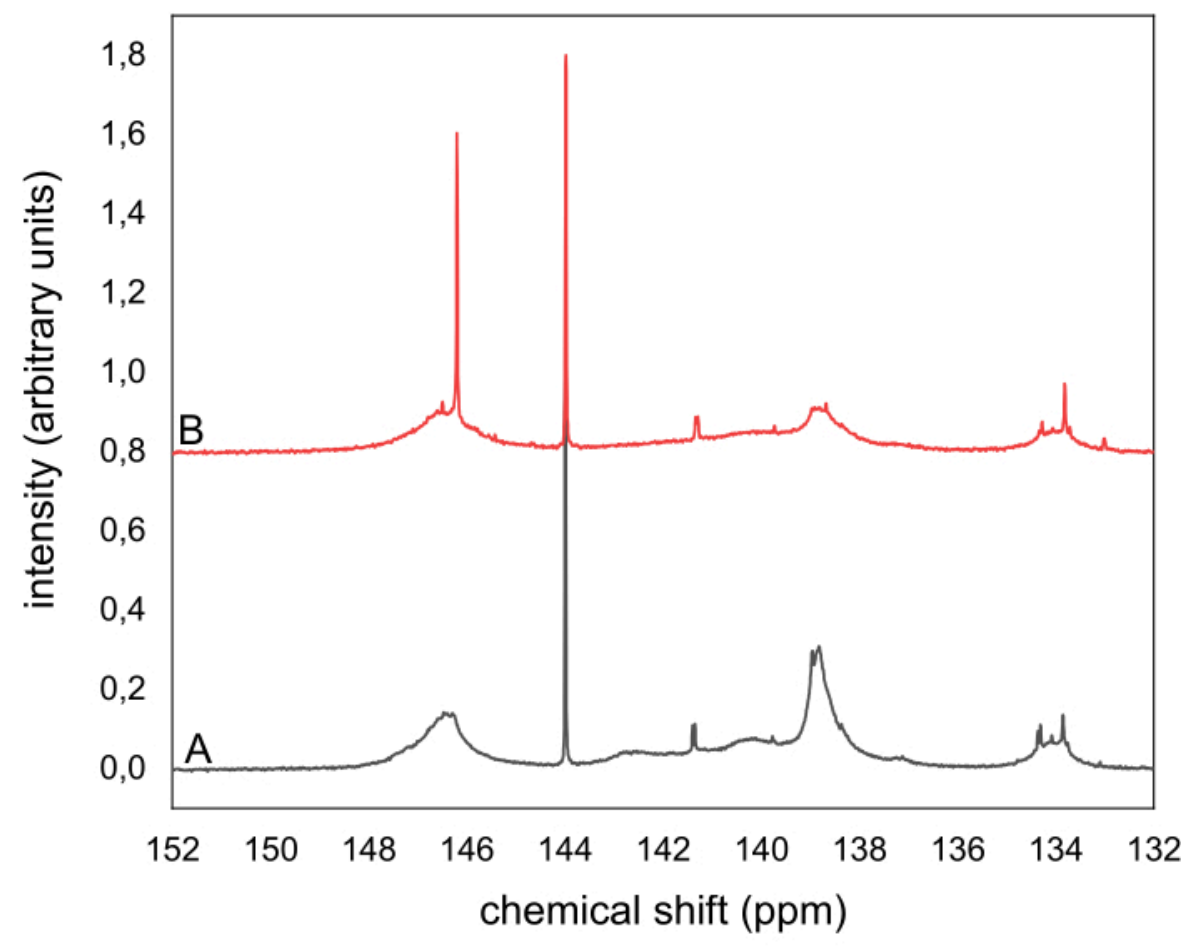

Figure 9: Quantitative ${ }^{31}$ P NMR spectra of the same Organosolv lignin derivatized using TMDP (A) Prior and (B) post its oxidation. The spectra were recorded using a 300 NMR spectrometer. Please click here to view a larger version of this figure.

In conclusion, this technique has all the attributes of being among the most essential and powerful tools when inquiries dealing with polyphenolic, $\mathrm{OH}$ bearing lignins, and tannins (and even synthetic polymers) $49,50,51$ need to be made in a variety of fields, ranging from chemistry to engineering, from biology to polymer, and pharmaceutical applications. 


\section{Disclosures}

Claudia Crestini and Dimitris S Argyropoulos ensure that all authors (C.C., N.P., and D.S.A.) have no conflicts of interest.

\section{Acknowledgments}

This work over the years has been supported by various financial awards that included organizations such as the Pulp and Paper Research Institute of Canada, McGill University Montreal, the Natural Sciences and Engineering Research Council of Canada, National Science Foundation USA, United States Department of agriculture, and the Solvay company.

\section{References}

1. Meng, X. et al. Determination of hydroxyl groups in biorefinery resources via quantitative $31 \mathrm{P}$ NMR spectroscopy. Nature Protocols. 14 (9), 2627-2647 (2019).

2. Anastas, P. T., Williamson, T. C. Green chemistry: An overview. Green Chemistry. 626, 1-17 (1996).

3. Anastas, P., Eghbali, N. Green chemistry: Principles and practice. Chemical Society Reviews. 39 (1), 301-312 (2010).

4. Collins, M. N. et al. Valorization of lignin in polymer and composite systems for advanced engineering applications - A review. International Journal of Biological Macromolecules. 131, 828-849 (2019).

5. Biorefinery: From Biomass to Chemicals and Fuels. Biorefinery. De Gruyter (2012).

6. Sannigrahi, P., Pu, Y., Ragauskas, A. Cellulosic biorefineries-unleashing lignin opportunities. Current Opinion in Environmental Sustainability. 2 (5), 383-393 (2010).
7. Lange, H., Decina, S., Crestini, C. Oxidative upgrade of lignin - Recent routes reviewed. European Polymer Journal. 49 (6), 1151-1173 (2013).

8. Glasser, W. G. Classification of lignin according to chemical and molecular structure. Lignin: Historical, Biological, and Materials Perspectives. 742, 216-238 (1999).

9. Kirk-Othmer. Concise Encyclopedia of Chemical Technology. 2 Volume Set, 5th Edition, Wiley (2004).

10. Lewis, N. G., Sarkanen, S. Preface. Lignin and Lignan Biosynthesis. 697, 9-11 (1998).

11. Adler, E. Lignin chemistry-past, present and future. Wood Science and Technology. 11 (3), 169-218 (1977).

12. Ragauskas, A. J. et al. Lignin valorization: Improving lignin processing in the biorefinery. Science. 344 (6185) (2014).

13. Crestini, C., Melone, F., Sette, M., Saladino, R. Milled wood lignin: A linear oligomer. Biomacromolecules. 12 (11), 3928-3935 (2011).

14. Guerra, A. et al. On the propensity of lignin to associate: A size exclusion chromatography study with lignin derivatives isolated from different plant species. Phytochemistry. 68 (20), 2570-2583 (2007).

15. Contreras, S., Gaspar, A. R., Guerra, A., Lucia, L. A., Argyropoulos, D. S. Propensity of lignin to associate: Light scattering photometry study with native lignins. Biomacromolecules. 9 (12), 3362-3369 (2008).

16. Gigli, M., Crestini, C. Fractionation of industrial lignins: opportunities and challenges. Green Chemistry. 22 (15), $4722-4746(2020)$.

17. Adler, E. Structural elements of lignin. Industrial \& Engineering Chemistry. 49 (9), 1377-1383 (1957). 
18. Bjorkman, A. Studies on finely divided wood. Part 1. Extraction of lignin with neutral solvents. Svensk Pappersit. 477-485 (1956).

19. Bjorkman, A. Studies on finely divided wood. Part 2. Extraction of lignin-carbohydrate compelexes with neutral solvents. Svensk Pappersit. 243-251 (1957).

20. Bjorkman, A. Studied on finely divided wood. Part 5. The effect of milling. Svensk Pappersit. 329-335 (1957).

21. Das, A. K., Islam, Md. N., Faruk, Md. O., Ashaduzzaman, Md., Dungani, R. Review on tannins: Extraction processes, applications and possibilities. South African Journal of Botany. 135, 58-70 (2020).

22. Laitila, J. E. Composition and evolution of oligomeric proanthocyanidin-malvidin glycoside adducts in commercial red wines. Food Chemistry. 340, 127905 (2021).

23. Covington, A. D., Wise, W. R. Tanning Chemistry. RSC Publishing (2019).

24. Tarabanko, V. E., Tarabanko, N. Catalytic oxidation of lignins into the aromatic aldehydes: General process trends and development prospects. International Journal of Molecular Sciences. 18 (11), 2421 (2017).

25. Guerra, A., Mendonça, R., Ferraz, A., Lu, F., Ralph, J. Structural characterization of lignin during pinus taeda wood treatment with ceriporiopsis subvermispora. Applied and Environmental Microbiology. 70 (7), 4073-4078 (2004).

26. Faix, O., Andersons, B., Zakis, G. Determination of carbonyl groups of six round robin lignins by modified oximation and FTIR spectroscopy. Holzforschung. 52 (3), 268-274 (1998).
27. Santos, R. B., Capanema, E. A., Balakshin, M. Y., Chang, H., Jameel, $H$. Lignin structural variation in hardwood species. Journal of Agricultural and Food Chemistry. 60 (19), 4923-4930 (2012).

28. Bose, S. K., Wilson, K. L., Hausch, D. L., Francis, R. C. Lignin analysis by permanganate oxidation. II. Lignins in Acidic Organosolv Pulps. Holzforschung. 53 (6), 603-610 (1999).

29. Harman-Ware, A. E. et al. A thioacidolysis method tailored for higher-throughput quantitative analysis of lignin monomers. Biotechnology Journal. 11 (10), 1268-1273 (2016).

30. Lupoi, J. S., Singh, S., Parthasarathi, R., Simmons, B. A., Henry, R. J. Recent innovations in analytical methods for the qualitative and quantitative assessment of lignin. Renewable and Sustainable Energy Reviews. 49, 871-906 (2015).

31. Lin, S. Y., Carlton, W. D. Methods in Lignin Chemistry. Springer, Berlin, Heidelberg (1992).

32. Lundquist, K. Proton (1H) NMR Spectroscopy. Methods in Lignin Chemistry. 242-249 (1992).

33. Robert, D. Carbon-13 nuclear magnetic resonance spectrometry. Methods in Lignin Chemistry. 250-273 (1992).

34. Li, S., Lundquist, K. A new method for the analysis of phenolic groups in lignins by ${ }^{1} \mathrm{H}$ NMR spectrometry. Nordic Pulp \& Paper Research Journal. 9 (3), 191-195 (1994).

35. Hallac, B. B., Pu, Y., Ragauskas, A. J. Chemical transformations of buddleja davidii lignin during ethanol organosolv pretreatment. Energy \& Fuels. 24 (4), 2723-2732 (2010). 
36. Sette, M., Wechselberger, R., Crestini, C. Elucidation of lignin structure by quantitative 2D NMR. Chemistry - $A$ European Journal. 17 (34), 9529-9535 (2011).

37. Sette, M., Lange, H., Crestini, C. Quantitative HSQC analyses of lignin: A practcal comparison. Computational and Structural Biotechnology Journal. 6 (7), e201303016 (2013).

38. Wroblewski, A. E., Lensink, C., Markuszewski, R., Verkade, J. G. Phosphorus-31 NMR spectroscopic analysis of coal pyrolysis condensates and extracts for heteroatom functionalities possessing labile hydrogen. Energy \& Fuels. 2 (6), 765-774 (1988).

39. Archipov, Y., Argyropoulos, D. S., Bolker, H. I., Heitner, C. ${ }^{31} \mathrm{P}$ NMR spectroscopy in wood chemistry. Part I. Model compounds. Journal of Wood Chemistry and Technology. 11 (2), 137-157 (1991).

40. Argyropoulos, D. S., Heitner, C., Morin, F. G. P NMR spectroscopy in wood chemistry - Part III. Solid state ${ }^{31} \mathrm{P}$ NMR of trimethyl phosphite derivatives of chromophores in mechanical pulp. Holzforschung - International Journal of the Biology, Chemistry, Physics and Technology of Wood. 46 (3), 211-218 (2009).

41. Argyropoulos, D. S., Heitner, C. ${ }^{31}$ P NMR spectroscopy in wood chemistry. Part VI. Solid state ${ }^{31} \mathrm{P}$ NMR of trimethyl phosphite derivatives of chromophores and carboxylic acids present in mechanical pulps; a method for the quantitative determination of ortho-quinones. Holzforschung. 48 (s1), 112-116 (1994).

42. Argyropoulos, D. S., Bolker, H. I., Heitner, C., Archipov, $\mathrm{Y} .{ }^{31} \mathrm{P}$ NMR spectroscopy in wood chemistry part $\mathrm{V}$. Qualitative analysis of lignin functional groups. Journal of Wood Chemistry and Technology. 13 (2), 187-212 (1993).

43. Argyropoulos, D. S., Bolker, H. I., Heitner, C., Archipov, Y. ${ }^{31} \mathrm{P}$ NMR spectroscopy in wood chemistry. Part IV. Lignin models: Spin lattice relaxation times and solvent effects in ${ }^{31}$ P NMR. Holzforschung. 47 (1), 50-56 (1993).

44. Granata, A., Argyropoulos, D. S. 2-Chloro-4,4,5,5tetramethyl-1,3,2-dioxaphospholane, a reagent for the accurate determination of the uncondensed and condensed phenolic moieties in lignins. Journal of Agricultural and Food Chemistry. 43 (6), 1538-1544 (1995).

45. Duval, A., Vilaplana, F., Crestini, C., Lawoko, M. Solvent screening for the fractionation of industrial kraft lignin. Holzforschung. 70 (1), 11-20 (2016).

46. Ben, H., Farrell III, J. R. In-depth investigation on quantitative characterization of pyrolysis oil by ${ }^{31} \mathrm{P} N M R$. RSC Advances. 6 (21), 17567-17573 (2016).

47. Goldschmid, O. Determination of phenolic hydroxyl content of lignin preparations by ultraviolet spectrophotometry. Analytical Chemistry. 26 (9), 1421-1423 (1954).

48. Ben, $\mathrm{H}$. et al. Characterization of whole biomasses in pyridine based ionic liquid at low temperature by ${ }^{31} \mathrm{P}$ NMR: An approach to quantitatively measure hydroxyl groups in biomass as their original structures. Frontiers in Energy Research. 6 (2018).

49. Debuissy, T., Pollet, E., Avérous, L. Synthesis of potentially biobased copolyesters based on adipic acid and butanediols: Kinetic study between 1,4- and 2,3butanediol and their influence on crystallization and thermal properties. Polymer. 99, 204-213 (2016). 
50. Debuissy, T., Pollet, E., Avérous, L. Synthesis and characterization of biobased poly(butylene succinateran-butylene adipate). Analysis of the compositiondependent physicochemical properties. European Polymer Journal. 87, 84-98 (2017).

51. Chan, K. P., Argyropoulos, D. S., White, D. M., Yeager, G. W., Hay, A. S. Facile quantitative analysis of hydroxyl end groups of Poly(2,6-dimethyl-1,4-phenylene oxide)s by ${ }^{31} \mathrm{P}$ NMR spectroscopy. Macromolecules. 27 (22), 6371-6375 (1994). 